\title{
Analysis of Particle Transfer by Periodic Lattice Modulation for Ultracold Fermionic Atom Systems in Three Dimensional Optical Lattice
}

\author{
Motoyoshi Inoue*, Yusuke Nakamura, and Yoshiya Yamanaka \\ Department of Electronic and Photonic Systems, Waseda University 3-4-1 Okubo \\ Shinjuku-ku Tokyo 169-8555, Japan
}

\begin{abstract}
We analyze a ultracold fermionic atom system in a three dimensional optical lattice with a confinement harmonic potential, using the Hubbard model, and time-dependent Gutzwiller variational approach for numerical calculation. Our study is focused on the time evolution of the particle transfer when the lattice potential is modulated by adding a periodic one. The choice of the parameters such as the modulation frequency and amplitude and the particle number affects the particle transfer. We calculate the time evolution of the variance in the particle distribution, and show its dependence on the parameters. The lattice modulation turns out to work effectively in order to control the particle transfer, and will be a useful method in experiments for fermionic atom systems.

KEYWORDS: optical lattice modulation, particle transfer dynamics, three dimensional inhomogeneous system, Fermi Hubbard model, Gutzwiller variational approach, insulator transition
\end{abstract}

\section{Introduction}

The ultracold fermionic atom system in an optical lattice attracts attention, since some intriguing phenomena such as Mott insulator and anti-ferromagnetism have been observed in the experiments and its analyses will bring hints to understand many problems in solid state physics. The systems, both bosonic and fermionic, are well described by the Hubbard model, whose numerical analysis are performed in various methods, i.e., Gutzwiller variational approach (GVA), ${ }^{1-3)}$ dynamical mean-field theory (DMFT), ${ }^{4-6)}$ density matrix renormalization group (DMRG) method, ${ }^{7-10)}$ quantum Monte Carlo (QMC) method ${ }^{11-13)}$ and so on.

To control the particle distribution of fermionic atoms in an optical lattice is not easy, since the transfer of fermionic atoms in an optical lattice is slower than that of bosonic one due to the Pauli blocking. The experiment to realize the Mott insulator transition in fermionic case is more difficult than those of bosonic ones in general. Actually it takes several tens of 
milliseconds to configure the ground state of Mott insulator in the bosonic experiment, ${ }^{14)}$ but several hundreds of milliseconds in the fermionic one. ${ }^{15)}$

As a promising way to manage to control the particle distribution of fermionic atoms in an optical lattice, the periodic lattice modulation, which means an addition of an oscillating lattice to the constant one, has been studied in the theoretical analysis for fermionic atom system using linear response ${ }^{7,11)}$ and in the experiment. ${ }^{16)}$ When the lattice modulation is under consideration, there are a few parameters, i.e., the modulation amplitude and frequency which can be varied easily in experiments and are expected to affect the transfer crucially. The previous studies show that the particle transfer is stimulated by the periodic lattice modulation, especially, for the resonant frequency of the lattice modulation and for the large modulation amplitude, though they are limited to the case close to the half-filling and to the homogeneous system without a confinement harmonic potential. The responses to the lattice modulation will be different for the homogeneous and inhomogeneous systems. The purpose of this paper is to analyze the response or particle transfer of fermionic atom system in a three dimensional optical lattice modulated by a periodic potential with a confinement harmonic potential. Since the experimental parameters are well controllable in ultracold atom systems, the analysis of the lattice modulation response in the inhomogeneous system will enable us to understand some new physical properties of complicated fermionic systems.

We analyze our three dimensional system, using the Hubbard model. The DMRG method for higher dimensional system remains to be established. The numerical calculations for the inhomogeneous three dimensional system are computationally expensive for the QMC or DMFT, so they are not realistic choices. The cost of numerical calculation in the GVA is lower than those in the other methods and reasonable, so GVA is the most suitable for our calculation. We numerically calculate the dynamical evolution under the time-dependent GVA. ${ }^{17,18)}$

We investigate the time evolution of the particle distribution, explicitly that of the variance in the particle distribution as an indicator of particle transfer, starting from some spatially inhomogeneous distributions and under the periodic lattice modulation The results are shown for various values of the parameters, the lattice modulation amplitude and frequency, the initial phase of the modulation and the total particle number. Finally we argue the choices of the parameter values to promote the particle transfer to the outer sites, and this knowledge will be helpful in designing experiments of fermionic systems.

This paper is structured as follows. In Sec. 2, we develop our theoretical approach using the Fermi Hubbard model and the Gutzwiller approximation. The section is separated into two parts, the Gutzwiller wave function (GWF) of the ground states and time-dependent 
GVA. The former is used for the initial condition in the latter, and the latter yields the time evolution of the system. The results of numerical calculations are presented in Sec. 3. Section 4 is devoted to summary and conclusion.

\section{Gutzwiller variational approach}

\subsection{Ground state}

In this subsection, we briefly review the Gutzwiller variational approach $(\mathrm{GVA})^{1-3)}$ to obtain the ground state of the Hubbard model for three dimensional system. The Hubbard Hamiltonian is

$$
\hat{H}=-J \sum_{\langle i, j\rangle, s} \hat{a}_{i, s}^{\dagger} \hat{a}_{j, s}+\sum_{i, s}\left(v_{i}-\mu\right) \hat{n}_{i, s}+U \sum_{i} \hat{n}_{i, \uparrow} \hat{n}_{i, \downarrow}, \quad \hat{n}_{i, s}=\hat{a}_{i, s}^{\dagger} \hat{a}_{i, s} .
$$

The first term is the hopping one, and there the hopping coefficient $J$ is independent both of the site indices $\boldsymbol{i}=\left(i_{x}, i_{y}, i_{z}\right)$ and the spin index $s=\uparrow, \downarrow$. The sum index $\langle\boldsymbol{i}, \boldsymbol{j}\rangle$ denotes the sum over the nearest neighbor sites. The second term represents the confinement potential and the chemical one, and the third one does the on-site interaction.

In GVA, the variational trial function, called the Gutzwiller wave function (GWF), is taken as

$$
\left|\Psi_{G}\right\rangle=\prod_{i} \hat{P}_{i}\left|\Psi_{0}\right\rangle=\prod_{i} \sum_{\Gamma} \lambda_{i, \Gamma} \hat{m}_{i, \Gamma}\left|\Psi_{0}\right\rangle,
$$

where $\hat{m}_{\boldsymbol{i}, \Gamma}=|\boldsymbol{i}, \Gamma\rangle\langle\boldsymbol{i}, \Gamma|$ is the projection operator of particle state in site $\boldsymbol{i}$, and $\Gamma$ stands for one of the four single site states $\{0, \uparrow, \downarrow, \uparrow \downarrow\}$. The key point of GVA is that there are two kinds of variational quantities, i.e., the parameter $\lambda_{i, \Gamma}$ and the wave function $\left|\Psi_{0}\right\rangle$. The former is chosen to minimize the total energy, while the latter is so to minimize the kinetic energy, as will be seen below. The expectation value of any product of operators, which is boson-like and local in site, with respect to $\left|\Psi_{0}\right\rangle$ is approximated (Gutzwiller approximation) as

$$
\left\langle\Psi_{0}\left|\hat{A}_{i} \hat{B}_{j}\right| \Psi_{0}\right\rangle=\left\langle\Psi_{0}\left|\hat{A}_{i}\right| \Psi_{0}\right\rangle\left\langle\Psi_{0}\left|\hat{B}_{j}\right| \Psi_{0}\right\rangle
$$

when $\boldsymbol{i} \neq \boldsymbol{j}$. It implies

$$
\left\langle\Psi_{G} \mid \Psi_{G}\right\rangle=\prod_{i}\left\langle\Psi_{0}\left|\hat{P}_{i}^{\dagger} \hat{P}_{i}\right| \Psi_{0}\right\rangle=\prod_{i}\left(\sum_{\Gamma} m_{i, \Gamma}\right),
$$

with

$$
m_{i, \Gamma}=\left|\lambda_{i, \Gamma}\right|^{2}\left\langle\Psi_{0}\left|\hat{m}_{i, \Gamma}\right| \Psi_{0}\right\rangle .
$$


For the normalization of $\left|\Psi_{G}\right\rangle$, we set

$$
\sum_{\Gamma} m_{i, \Gamma}=1
$$

It is also assumed that $\left|\Psi_{0}\right\rangle$ is not spin entangled,

$$
\left|\Psi_{0}\right\rangle=\left|\psi_{\uparrow}\right\rangle \otimes\left|\psi_{\downarrow}\right\rangle
$$

Then from the Gutzwiller approximation (3) and the identity

$$
\hat{P}_{i}^{\dagger} \hat{a}_{i, s} \hat{P}_{i}=\left\{\lambda_{i, s} \lambda_{i, 0}^{*}\left(1-\hat{n}_{i, \bar{s}}\right)+\lambda_{i, \uparrow \downarrow} \lambda_{i, \bar{s}}^{*} \hat{n}_{i, \bar{s}}\right\} \hat{a}_{i, s},
$$

where $\bar{s}$ denotes the opposite spin to $s$, we obtain the expectation value of the total energy,

$$
\left\langle\Psi_{G}|\hat{H}| \Psi_{G}\right\rangle=-J \sum_{\langle i, j\rangle, s} Z_{i, s}^{*} Z_{j, s}\left\langle\Psi_{0}\left|\hat{a}_{i, s}^{\dagger} \hat{a}_{j, s}\right| \Psi_{0}\right\rangle+\sum_{i}\left\{\left(v_{i}-\mu\right)\left(m_{i, \uparrow}+m_{i, \downarrow}+2 m_{i, \uparrow \downarrow}\right)+U m_{i, \uparrow \downarrow}\right\},
$$

with

$$
Z_{i, s}=\lambda_{i, s} \lambda_{i, 0}^{*}\left(1-n_{i, \bar{s}}^{0}\right)+\lambda_{i, \uparrow \downarrow} \lambda_{i, \bar{s}}^{*} n_{i, \bar{s}}^{0}, \quad n_{i, s}^{0}=\left\langle\Psi_{0}\left|\hat{n}_{i, s}\right| \Psi_{0}\right\rangle
$$

This specific form of the total energy makes it possible to fix the variational parameter $\lambda_{i, s}$ to be real without loss of generality. For convenience we take the real parameter $m_{i, \Gamma}$ as the variational parameter instead of $\lambda_{i, s}$, both are related to each other by Eq. (5). The quantity $Z_{i, s}$ is rewritten as

$$
Z_{i, s}=\sqrt{\frac{m_{i, 0} m_{i, s}}{n_{i, s}^{0}\left(1-n_{i, s}^{0}\right)}}+\sqrt{\frac{m_{i, \bar{s}} m_{i, \uparrow \downarrow}}{n_{i, s}^{0}\left(1-n_{i, s}^{0}\right)}} .
$$

The variational wave function $\left|\Psi_{0}\right\rangle$ is determined by the requirement that it should minimize the kinetic energy,

$$
\left\langle\Psi_{G}\left|\hat{H}_{J}\right| \Psi_{G}\right\rangle=-J \sum_{\langle i, j\rangle, s} Z_{i, s} Z_{j, s}\left\langle\Psi_{0}\left|\hat{a}_{i, s}^{\dagger} \hat{a}_{j, s}\right| \Psi_{0}\right\rangle .
$$

This is accomplished by diagonalizing the effective hopping Hamiltonian

$$
\hat{H}_{\mathrm{J}}=\sum_{s} \hat{H}_{\mathrm{J}, s}=-J \sum_{\langle i, j\rangle, s} Z_{\boldsymbol{i}, s} Z_{\boldsymbol{j}, s} \hat{a}_{\boldsymbol{i}, s}^{\dagger} \hat{a}_{\boldsymbol{j}, s} .
$$

Because there is no cross-term of up- and down-spins in Eq. (13), $\left|\Psi_{0}\right\rangle$ is given by the direct product of the spin states, which is consistent with the assumption Eq. (7). The coefficient hopping Hamiltonian $\hat{H}_{\mathrm{J}, s}$ is diagonalized by the unitary transformation $\alpha_{p, s}=\sum_{i} U_{p, i, s} a_{i, s}$ as

$$
\hat{H}_{\mathrm{J}, s}=\sum_{p} \varepsilon_{p, s} \hat{\alpha}_{p, s}^{\dagger} \hat{\alpha}_{p, s}
$$


and the ground state $\left|\Psi_{0}\right\rangle$ is determined as

$$
\left|\Psi_{0}\right\rangle=\left(\prod_{\boldsymbol{p}}^{\varepsilon_{p, \uparrow}<0} \alpha_{p, \uparrow}^{\dagger}\right)|0\rangle_{\uparrow} \otimes\left(\prod_{p}^{\varepsilon_{p, \downarrow}<0} \alpha_{p, \downarrow}^{\dagger}\right)|0\rangle_{\downarrow}
$$

where $|0\rangle_{s}$ is the vacuum of $\alpha_{p, s}$. Thus we obtain

$$
\left\langle\Psi_{0}\left|\hat{a}_{i, s}^{\dagger} \hat{a}_{j, s}\right| \Psi_{0}\right\rangle=\sum_{s} \sum_{p}^{\varepsilon_{p, s}<0} U_{p, i, s} U_{p, j, s} .
$$

The quantity $Z_{i, s}$ and consequently the unitary matrix $U_{p, i, s}$ are functions of $m_{i, \Gamma}$, which in turn depends on $\left|\Psi_{0}\right\rangle$ as in Eq. (5). So the variational quantities $m_{i, \Gamma}$ and $\left|\Psi_{0}\right\rangle$ have to be determined in a self-consistent manner.

\subsection{Dynamics}

We next review the time-dependent GVA. ${ }^{17,18)}$ To describe the dynamics of the present system, the stationary GWF is extended straightforwardly to the time-dependent one by making the variational parameter $\lambda_{i, \Gamma}$ complex and time-dependent,

$$
\left|\Psi_{G}(t)\right\rangle=\prod_{i} \sum_{\Gamma} \lambda_{i, \Gamma}(t) \hat{m}_{i, \Gamma}\left|\Psi_{0}\right\rangle
$$

The equation of time evolution is derived by the variational principle for the action,

$$
S=\int d t\left\langle\Psi_{G}(t)\left|i \hbar \frac{d}{d t}-\hat{H}\right| \Psi_{G}(t)\right\rangle .
$$

The integrand is manipulated as

$$
\left\langle\Psi_{G}(t)\left|i \hbar \frac{d}{d t}-\hat{H}\right| \Psi_{G}(t)\right\rangle=\hbar \sum_{i, \Gamma}\left(\prod_{j \neq i} \sum_{\Gamma^{\prime}} m_{j, \Gamma^{\prime}}(t)\right)\left(\dot{\phi}_{i, \Gamma}(t) m_{i, \Gamma}+\frac{i}{2} \dot{m}_{i, \Gamma}(t)\right)-E\left[m_{i, \Gamma}, \phi_{i, \Gamma}\right],
$$

with $\phi_{i, \Gamma}(t)=-\arg \lambda_{i, \Gamma}(t), \quad m_{i, \Gamma}(t)=\left|\lambda_{i, \Gamma}(t)\right|^{2}\left\langle\Psi_{0}\left|\hat{m}_{i, \Gamma}\right| \Psi_{0}\right\rangle$, and $E\left[m_{i, \Gamma}, \phi_{i, \Gamma}, t\right]=$ $\left\langle\Psi_{G}(t)|\hat{H}| \Psi_{G}(t)\right\rangle$. For the conservation of the normalization $\left\langle\Psi_{G}(t) \mid \Psi_{G}(t)\right\rangle=1$, we require

$$
\sum_{\Gamma} m_{i, \Gamma}(t)=1
$$

whose consistency will be confirmed later. Then the action is simplified as

$$
S=\int d t\left(\sum_{i, \Gamma} \hbar \dot{\phi}_{i, \Gamma}(t) m_{i, \Gamma}(t)-E\left[m_{i, \Gamma}, \phi_{i, \Gamma}\right]\right),
$$

which implies that $\phi_{i, \Gamma}$ and $m_{i, \Gamma}$ are a pair of canonical variables. Their equations of motion are given by

$$
\hbar \dot{m}_{i, \Gamma}=-\frac{\partial E}{\partial \phi_{i, \Gamma}}, \quad \hbar \dot{\phi}_{i, \Gamma}=\frac{\partial E}{\partial m_{i, \Gamma}} .
$$

The total energy is expressed in the same form as Eq. (9) but the coefficients become 
complex and time-dependent:

$$
\begin{aligned}
E\left[m_{i, \Gamma}, \phi_{i, \Gamma}\right]= & -J \sum_{\langle i, j\rangle, s} Z_{i, s}^{*}(t) Z_{j, s}(t)\left\langle\Psi_{0}\left|\hat{a}_{i, s}^{\dagger} \hat{a}_{j, s}\right| \Psi_{0}\right\rangle \\
& +\sum_{i}\left\{\left(v_{i}-\mu\right)\left(m_{i, \uparrow}(t)+m_{i, \downarrow}(t)+2 m_{i, \uparrow \downarrow}(t)\right)+U m_{i, \uparrow \downarrow}(t)\right\} .
\end{aligned}
$$

with

$$
Z_{i, s}(t)=\sqrt{\frac{m_{i, 0}(t) m_{i, s}(t)}{n_{i, s}^{0}\left(1-n_{i, s}^{0}\right)}} e^{i\left(\phi_{i, 0}(t)-\phi_{i, s}(t)\right)}+\sqrt{\frac{m_{i, \bar{s}}(t) m_{i, \uparrow \downarrow}(t)}{n_{i, s}^{0}\left(1-n_{i, s}^{0}\right)}} e^{i\left(\phi_{i, \bar{s}}(t)-\phi_{i, \uparrow \downarrow}(t)\right)} .
$$

Because of the relations

$$
\frac{\partial Z_{i, s}}{\partial \phi_{i, 0}}+\frac{\partial Z_{i, s}}{\partial \phi_{i, s}}=0, \quad \frac{\partial Z_{i, s}}{\partial \phi_{i, \uparrow \downarrow}}+\frac{\partial Z_{i, s}}{\partial \phi_{i, \bar{s}}}=0,
$$

we obtain

$$
\sum_{\Gamma} \frac{\partial E}{\partial \phi_{i, \Gamma}}=0
$$

This, with Eq. (22), leads to Eq. (20), and the consistency of Eq. (20) has been checked. We can also confirm the energy conservation

$$
\frac{d}{d t} E\left[m_{i, \Gamma}, \phi_{i, \Gamma}\right]=\sum_{i, \Gamma}\left(\frac{\partial E}{\partial m_{i, \Gamma}} \dot{m}_{i, \Gamma}+\frac{\partial E}{\partial \phi_{i, \Gamma}} \dot{\phi}_{i, \Gamma}\right)=0 .
$$

\section{Numerical Results}

In this section, we present the results of numerical calculations according to the variational formulations in the previous section. Our main interest is in the dynamics of particle transfer, and we devote ourselves below to study it under the periodic lattice modulation, meaning that an oscillating sinusoidal lattice potential is added for $t>0$ to the constant one. Equation (22) is solved numerically, and it will be shown how the particles are transferred for various values of the amplitude and frequency of the lattice modulation.

\subsection{Parameters}

In our numerical calculations below, the spatial site number is $18^{3}$. The confinement harmonic potential is assumed to be isotropic with its center at that of the lattice system, $\boldsymbol{i}_{0}=\frac{18-1}{2}(1,1,1)=(8.5,8.5,8.5)$, and to have the fixed strength,

$$
v_{i}=0.2 E_{\mathrm{R}}\left|\boldsymbol{i}-\boldsymbol{i}_{0}\right|^{2}
$$

where $E_{\mathrm{R}}=h^{2} / 2 m \lambda^{2}$ is the recoil energy and $\lambda$ is the wavelength of the laser light, corresponding to a lattice period $a=\lambda / 2$. The constant lattice amplitude before the lattice potential is modulated is put as $\bar{V}_{0}=6.5 E_{\mathrm{R}}$, and this value is not so large that the stable insulator could 

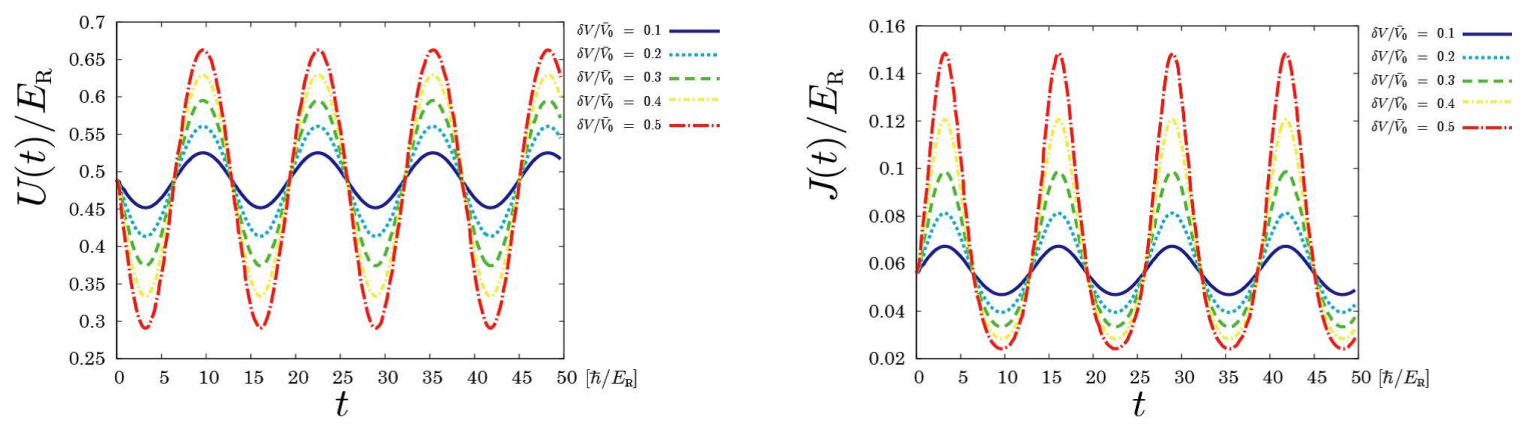

Fig. 1. Temporal behaviors of $U(t)$ and $J(t)$ for the modulation amplitude $\delta V / \bar{V}_{0}=0,0.1,0,2,0.3,0.4$, and 0.5 . The period of the oscillation is $12.9 \hbar / E_{\mathrm{R}}$.

be formed. The s-wave scattering length is $a_{s}=240 a_{0}$, where $a_{0}$ is Bohr radius. We quote these parameters from the experimental ones. ${ }^{15}$

The lattice is modulated as a sudden switch-on of the sinusoidal potential at $t=0$ :

$$
V_{0}(t)= \begin{cases}\bar{V}_{0} & t<0, \\ \bar{V}_{0}-\delta V \sin [\omega t+\theta] & t>0,\end{cases}
$$

where $\delta V$ and $\omega$ are referred to the modulation amplitude and frequency, respectively. It is expected that the frequency resonant with the interaction energy invokes the particle transport most effectively. Note that the parameters of the Hubbard model, $J(t)$ and $U(t)$, are timedependent as follows, ${ }^{19)}$ since they are functions of $V_{0}(t)$,

$$
\begin{aligned}
U(t) & =4 \sqrt{2 \pi}\left(a_{s} / \lambda\right)(\tilde{v}(t))^{3 / 4} E_{\mathrm{R}}, \\
J(t) & =4 / \sqrt{\pi}(\tilde{v}(t))^{3 / 4} \exp \left[-2(\tilde{v}(t))^{1 / 2}\right] E_{\mathrm{R}},
\end{aligned}
$$

with $\tilde{v}(t)=V_{0}(t) / E_{\mathrm{R}}$. Though $U(t)$ is time-dependent [see Eq.(30)], we define the resonant frequency $\omega_{\mathrm{re}}=\bar{U} / \hbar$, using the mean interaction strength $\bar{U}=U(t)$ for $t<0$. The phase in Eq. (29) is taken either $\theta=0$ or $\pi$. In this paper, we calculate for various strengths of the modulation amplitude, $\delta V / \bar{V}_{0}=0.1,0,2,0.3,0.4$, and 0.5 , and for various modulation frequencies $\omega / \omega_{\text {re }}=0.5,0.8,1.0,1.5,2.0$, and 2.5 .

The temporal behaviors of $U(t)$ and $J(t)$ in Eqs. (30) and (31) are plotted in Fig. 1. The hopping coefficient $J(t)$ behaves more intricately than the interaction one $U(t)$. This is due to the presence of the factor $\exp \left[-2(\tilde{v}(t))^{1 / 2}\right]$ in the former. We remark that the local maximum value of $J(t)$ is larger for larger $\delta V$, though it behaves almost sinusoidally for small $\delta V$, reflecting $(\tilde{v}(t))^{3 / 4}$.

The initial states at $t=0$ are the ground ones under the lattice potential $\bar{V}_{0}$, and their 

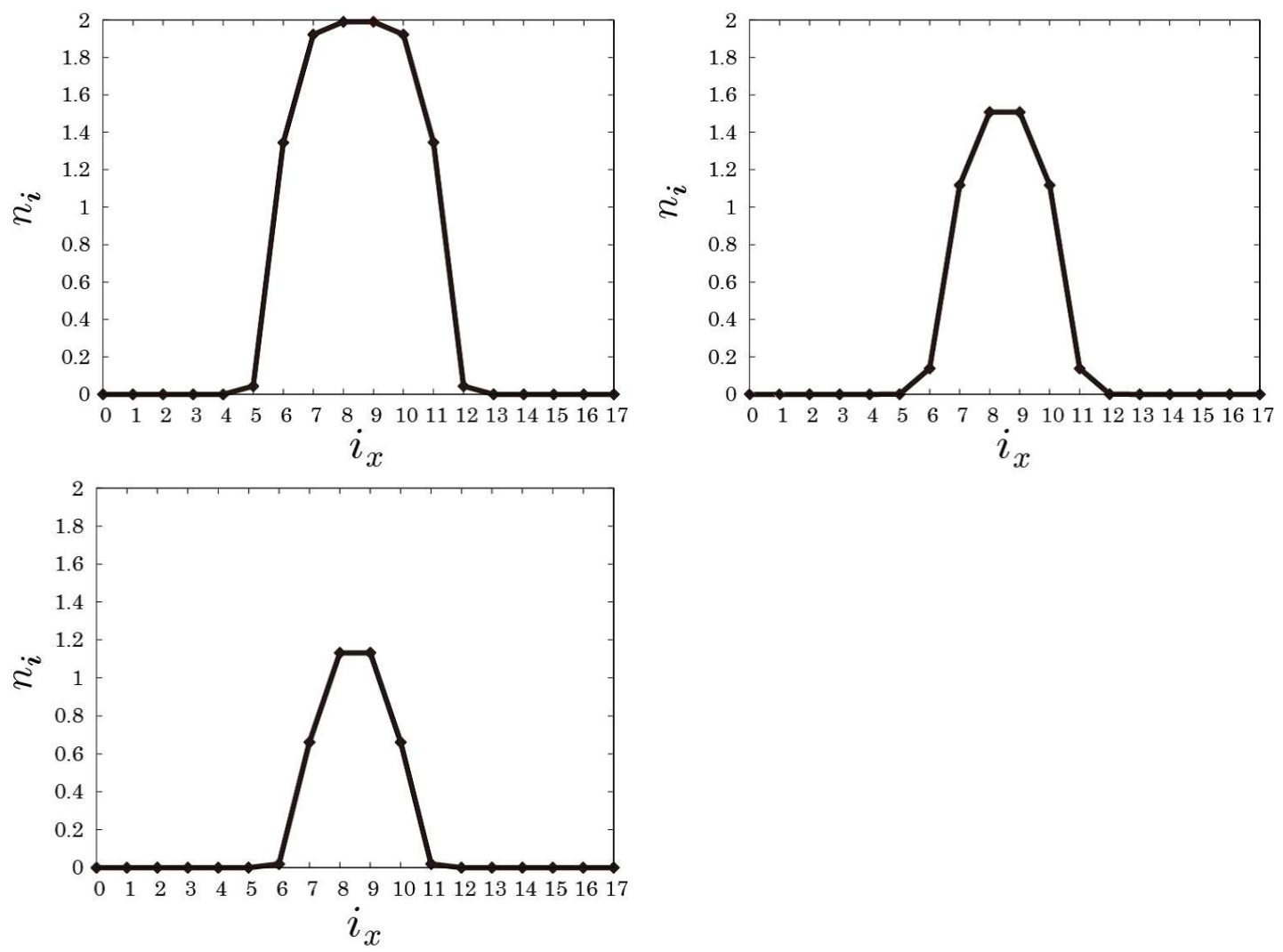

Fig. 2. Profiles of initial states: The particle number distributions are shown for $x$-axis at $i_{y}=i_{z}=9$. The particle number at site $i$ is $n_{i}=\left\langle\Psi_{G}\left|\hat{n}_{i, \uparrow}+\hat{n}_{i, \downarrow}\right| \Psi_{G}\right\rangle$. Figures (a), (b) and (c) correspond to $N=200,60,30$, respectively.

profiles are shown for the particle number $N=\sum_{i, s}\left\langle\Psi_{G}\left|\hat{a}_{i, s}^{\dagger} \hat{a}_{i, s}\right| \Psi_{G}\right\rangle=200,60,30$ in Fig. 2. In our simulations below, we also prepare the initial states for $N=100,40$. The responses for the various particle number states are compared in Subsec. 3.3.

Let us consider the variance in the particle distribution $\sigma^{2}(t)$,

$$
\sigma^{2}(t)=\frac{1}{N} \sum_{i, s}\left(\left|\boldsymbol{i}-\boldsymbol{i}_{0}\right| n_{i, s}(t)-\frac{1}{N} \sum_{\boldsymbol{i}^{\prime}, s^{\prime}}\left|\boldsymbol{i}^{\prime}-\boldsymbol{i}_{0}\right| n_{\boldsymbol{i}^{\prime}, s^{\prime}}(t)\right)^{2} .
$$

The initial variances are 5.65 for $N=200$ and 2.55 for $N=30$, respectively. Generally speaking, the increase in the variance indicates that the particles are tended to be transferred to the outer sites, while its decrease implies the particle transfer to the inner sites. We will use the variance $\sigma^{2}(t)$ as a measure of the particle transfer.

\subsection{Responses to the lattice modulation with various frequencies}

First, we show the time evolution of the variances in the particle distribution for various modulation frequencies, fixing the modulation amplitude and the initial phase. The initial 

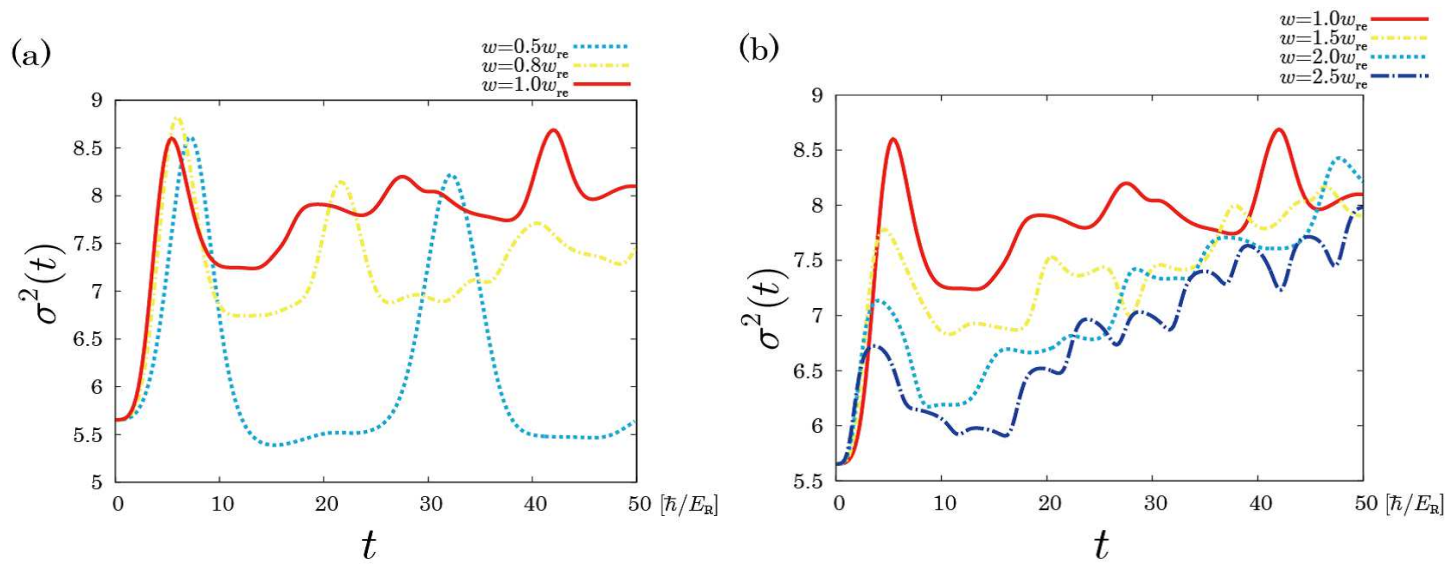

Fig. 3. Time evolution of the variances in the particle distribution for various modulation frequencies with the fixed modulation amplitude $\delta V / \bar{V}_{0}=0.5$ and initial phase $\theta=0$ : (a) $\omega / \omega_{\text {re }}=0.5,0.8,1.0$, (b) $\omega / \omega_{\text {re }}=$ $1.0,1.5,2.0,2.5$.

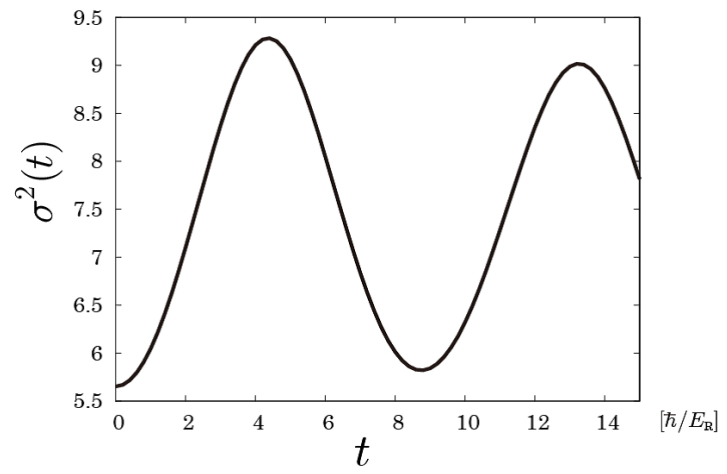

Fig. 4. Time evolution of the variances in the particle distribution after a sudden change of the lattice amplitude from $\bar{V}_{0}=6.5$ to $\bar{V}_{0}=3.25$ at $t=0$ with $\delta V=0$ and $N=200$.

state is that in Fig. 2 (a) $(N=200)$. The results are shown for lower frequencies in Fig. 3 (a) and for higher ones in Fig. 3 (b), separately. The fact that the variance $\sigma^{2}(t)$ becomes large in general indicates that the particles are transferred to the outer sites, responding to the lattice modulation. This is the most significant at the resonant frequency, though it takes place even at non-resonant frequencies.

We see in Fig. 3 (a) that the structure of a peak and a plateau is repeated for the offresonant frequencies $\left(\omega=0.8\right.$ and $\left.0.5 \omega_{\text {re }}\right)$. The time intervals between their first and second peaks are roughly the respective modulation period $\tau$, which are $\tau=16.1$ and $25.8 \hbar / E_{\mathrm{R}}$ corresponding $0.8 \omega_{\text {re }}$ and $0.5 \omega_{\text {re }}$, respectively. In order to interpret this, we give the temporal behavior of the variance after the lattice amplitude $\bar{V}_{0}$ is suddenly reduced to a half at $t=0$ (without the lattice modulation), which is illustrated in Fig. 4. There the oscillation is under- 


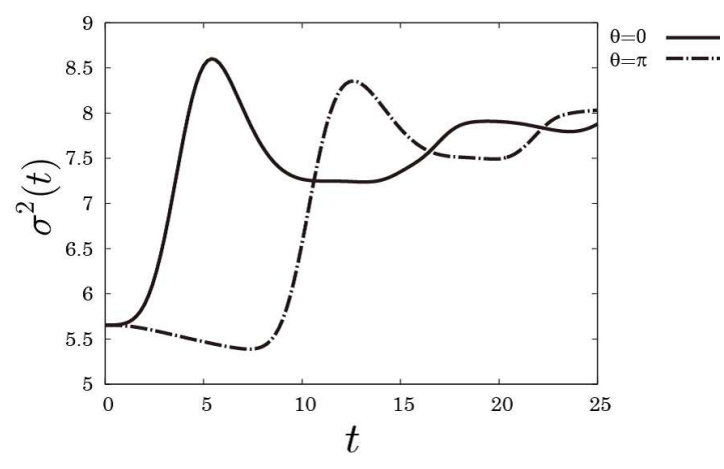

Fig. 5. The time evolution of the variances for the initial phases of the lattice modulation $\theta=0$ and $\pi$ with $\omega / \omega_{\text {re }}=1.0, \delta V / \bar{V}_{0}=0.5$ and $N=200$.

stood as follows: Due to the sudden decrease of the lattice potential height, it is easier for the particles to hop to the neighboring sites, and at first the particles tend to hop to the outer neighboring sites, since the particle population is higher at the inner sites, and then go back to the inner ones. It is difficult to compute the period of this oscillatory behavior, denoted by $T$, analytically, because many factors take part in it. The numerical calculation in Fig. 4 shows $T \simeq 8.8 \hbar / E_{\mathrm{R}}$. For $\omega=0.8$ and $0.5 \omega_{\mathrm{re}}$, the first oscillation peak appears as in Fig. 4 , but then the lattice potential $V_{0}(t)$ becomes higher than $\bar{V}_{0}$ and approaches to its maximum value $9.75 E_{\mathrm{R}}$ for which the lattice system is in the stable insulator. Therefore, the hopping to the neighboring sites is suppressed extremely, which explains the plateau. On the other hand, in case of $\omega=1.0 \omega_{\mathrm{re}}$, giving $\tau=12.9 \hbar / E_{\mathrm{R}}, T$ and $\tau$ are of the same order, and the suppression of the hopping does not last long so that no clear plateau is seen.

In Fig. 3 (b), the peak value of the first oscillation is the largest for the resonant frequency and becomes smaller as the frequency is higher. The variance continues to increase with the oscillation in all the cases, implying that the particles mainly moves to the outer sites and scarcely returns towards the center of the lattice system.

The time evolution of the variance $\sigma^{2}(t)$ should be compared for different $\theta$, concretely $\theta=0$ and $\pi$, and the result is depicted in Fig. 5. As is expected, both behave differently around the initial time but the difference gradually decreases as $t$ goes.

In summary of this subsection, the modulation frequency is crucial for the particle transfer. The transfer proceeds for the resonant frequency and higher ones, and is the most efficient and rapid for the resonant one. The intrinsic oscillation period $T$ after a sudden decrease in the lattice height is an essential parameter to inhibit the transfer for lower frequencies. The initial phase $\theta$ affects behaviors only for a short interval from the initial time. 


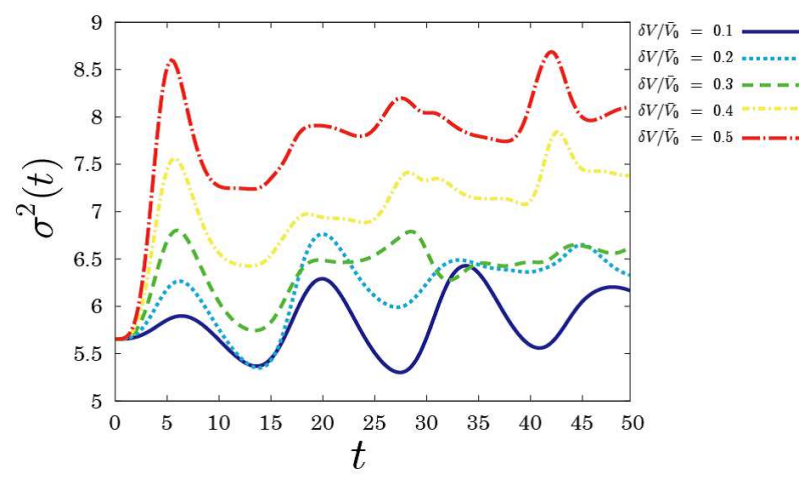

Fig. 6. Time evolution of the variance in the particle number distribution for various lattice amplitude $\delta V / \bar{V}_{0}=$ $0.1,0,2,0.3,0.4$, and 0.5 with $\omega / \omega_{\text {re }}=1.0, \theta=0$ and $N=200$.

\subsection{Responses to the lattice modulation with various amplitudes at the resonant frequency}

Next, let us see how the response depends on the modulation amplitude $\delta V$. The time evolution of the variance in the particle number distribution for various values of $\delta V$ at the resonant frequency is depicted in Fig. 6. It is seen from Fig. 6 that the variance follows a simple harmonic-like oscillation when $\delta V$ is small and that for the larger $\delta V$ it oscillates only for half a period and then increases in a stepwise pattern.

The particle hopping is suppressed when the lattice amplitude is so large that the stable insulator is formed, but is stimulated when the lattice amplitude is not so large. The value of the mean amplitude $\bar{V}_{0}=6.5 E_{\mathrm{R}}$ is not large, and the particles hop to the outer sites and then to the inner ones periodically for the lower $\delta V$. For the higher $\delta V$, the particle hopping is suppressed as $V_{0}(t)$ reaches its maximum, though it is stimulated around the minimal value of $V_{0}(t)$. The stimulation and suppression become more intensive as $\delta V$ becomes larger. This explains the complicated behaviors of the variances for $\delta V / \bar{V}_{0}=0.5$ and 0.4 in Fig. 6.

\subsection{Dependence of the response on particle number}

Finally we see how the response depends on the particle number. The time evolution of the variance in particle number distribution for various particle number $N$ with $\delta V=0.5 \bar{V}_{0}$ and on the resonant frequency $\omega=\omega_{\text {re }}$ is shown in Fig. 7. The variance follows a simple harmonic-like oscillation when the particle number is small $(N=30)$, and oscillates only for half a period and then increases in a stepwise pattern for the large particle number such as for $N=200$. This is explained as follows: the lattice system becomes a stable insulator more likely due to the on-site particle interaction and the Pauli blocking as the particle population is higher. For the interval in which $V_{0}(t)$ is large and the stable insulator is formed, the hopping to the neighboring sites is suppressed and the variance does not change, meaning the plateau 


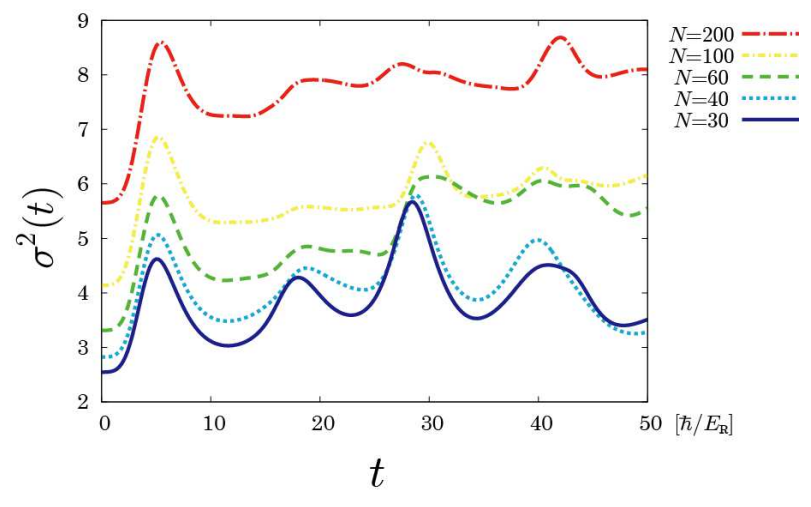

Fig. 7. Time evolution of the variance in the particle number distribution for various particle number $N=$ $200,100,60,40$, and 30 with $\omega / \omega_{\text {re }}=1.0, \theta=0$, and $\delta V / \bar{V}_{0}=0.5$.

structure. In contrast, the stable insulator is formed hardly for the lower particle population, and the hopping to the neighboring sites is scarcely suppressed for the large value of $V_{0}(t)$, and the variance change in a harmonic-like oscillation.

\section{Summary and Conclusion}

We consider the ultracold fermionic atom system in a three dimensional optical lattice with a confinement harmonic potential, and analyze it by use of the Hubbard model. Our study is focused on the dynamics of the particle transfer. Our numerical calculations are performed in the Gutzwiller variational approach, because the cost of calculation is low for higher dimensional and time-dependent systems. The lattice modulation frequency is crucial for the particle transfer.

We calculate the variance in the particle distribution, which is a simple quantity and nevertheless indicates the particle transfer properly. The results of our numerical calculations are summarized as follows: The variance changes most remarkably on the resonant modulation frequency, as quantum transitions are enhanced then. On the premise that the mean lattice amplitude $\bar{V}_{0}$ is close to the boundary value at which the stable insulator is formed, the particles are transferred to the outer sites for the resonant and higher frequencies. For the lower frequencies, the hopping of the particle to the neighboring sites is suppressed due to the stage of the stable insulator and the particle transfer is not simulated. The effect of the initial phase of the lattice modulation is restrictive. The modulation amplitude $\delta V$ is an important parameter. While the variance only oscillates for the lower $\delta V$, it continues to increases in a stepwise pattern, leading to the large particle transfer. As the total number of atoms $N$ is larger, that is, the particle population is higher, a stable insulator is formed more easily. This implies that 
the particle transfer is stimulated for the larger $N$, similarly as it is so for the larger $\delta V$.

In conclusion, it is useful to apply the lattice modulation to the fermionic system in a three dimensional optical lattice with a confinement harmonic potential in order to give rise to the particle transfer. The lattice modulation method is quite effective, when the modulation frequency is set to the resonant one, and the modulation amplitude and the particle number are large. The particle transfer can be controlled by the setup conditions on the parameters of the lattice modulation, and their proper management makes it possible to do more complicated experiments of fermionic systems in an optical lattice under control. 


\section{References}

1) J. Bünemann and W. Weber: Phys. Rev. B 57 (1998) 6896.

2) X. Y. Deng, L. Wang, X. Dai, and Z. Fang: Phys. Rev. B 79 (2009) 075114.

3) J. Q. Qi, L. Wang, and X. Dai: Chin. Phys. Lett. 27 (2010) 083102.

4) A. Georges, G. Kotliar, W. Krauth, and M. J. Rozenberg: Rev. Mod. Phys. 68 (1996) 13.

5) M. Snoek, I. Titvinidze, C. Töke, K. Byczuk, and W. Hofstetter: New J. Phys. 10 (2008) 093008.

6) E. A. Winograd, R. Chitra, and M. J. Rozenberg: Phys. Rev. B 86 (2012) 195118.

7) C. Kollath, A. Iucci, I. P. McCulloch, and T. Giamarchi: Phys. Rev. A 74 (2006) 041604.

8) T. Yamashita and N. Kawakami: Phys. Rev. A 74 (2006) 063624.

9) S. R. Manmana, S. Wesse, R. M. Noack, and A. Muramatsu: Phys. Rev. B 79 (2009) 155104.

10) J. W. Huo, W. Chen, U. Schollwöck, M. Troyer, and F. C. Zhang: Phys. Rev. A 86 (2012) 033611.

11) Z. Xu, S. Chiesa, S. Yang, S. Q. Su, D. E. Sheehy, J. Moreno, R. T. Scalettar, and M. Jarrell: Phys. Rev. A 84 (2011) 021607.

12) R. Blankenbecler, D.J.Scalapino and R. L. Sugar: Phys. Rev. D 24 (1981) 2278.

13) E. V. Gorelik and N. Blümer: Phys. Rev. A 80 (2009) 051602.

14) M. Greiner, O. Mandel, T. Esslinger, T. W. HäEnsch, and I. Bloch: Nature 415 (2002) 39.

15) R. Jöndens, N. Strohmaier, K. Günter, H. Moritz and T. Esslinger: Nature 455 (2008) 204.

16) D. Greif, L. Tarruell, T. Uehlinger, R. Jördens, and T. Esslinger: Phys. Rev. Lett. 106 (2011) 145302.

17) M. Schiró and M. Fabrizio: Phys. Rev. B. 83 (2011) 165105.

18) N. Lanatá and H. U. R. Strand: Phys. Rev. B 86 (2012) 115310.

19) W. Zwerger: J. Opt. B: Quantum Semiclass. Opt. 5 (2011) S9. 